\title{
Immune thrombocytopenic purpura risk by live, inactivated and simultaneous vaccinations among Japanese adults, children and infants: a matched case-control study
}

\author{
Hiroshi Yokomichi ${ }^{1}$ (1) $\cdot$ Keiko Tanaka-Taya ${ }^{2} \cdot$ Rie Koshida $^{3} \cdot$ Takashi Nakano $^{4} \cdot$ Yoshinori Yasui $^{5} \cdot$ Masaaki Mori $^{6}$. \\ Yuka Ando $^{7} \cdot$ Saeko Morino ${ }^{2} \cdot$ Hideo Okuno $^{2} \cdot$ Hiroshi Satoh $^{2} \cdot$ Satoru Arai ${ }^{2} \cdot$ Mie Mochizuki $^{8} \cdot$ Zentaro Yamagata $^{1}$
}

Received: 11 November 2019 / Revised: 25 March 2020 / Accepted: 25 March 2020 / Published online: 6 April 2020

(C) Japanese Society of Hematology 2020

\begin{abstract}
This case-control study investigated immune thrombocytopenic purpura (ITP) risk following live, inactivated, and simultaneous vaccination, with a focus on infants aged $<2$ years. We matched case patients with ITP to one or two control patients with other diseases by institution, hospital visit timing, sex, and age. We calculated McNemar's pairwise odds ratios (ORs [95\% confidence interval]) with 114 case-control pairs. The case group had $27(44 \%)$ males and $22(35 \%)$ infants, and the control group included $49(43 \%)$ males and $42(37 \%)$ infants. For all age groups, the McNemar's OR for ITP occurrence was $1.80(0.54-6.84, p=0.64)$ for all vaccines. Among infants, these were $1.50(0.17-18.0, p=0.50)$ for all vaccines, 2.00 $(0.29-22.1, p=0.67)$ for live vaccines, and $1.00(0.01-78.5, p=0.50)$ for inactivated vaccines. Sex-adjusted common ORs for simultaneous vaccination were $1.52(0.45-5.21, p=0.71)$ for all vaccines, $1.83(0.44-7.59, p=0.40)$ for inactivated vaccines only, and $1.36(0.29-6.30, p=0.69)$ for mixed live and inactivated vaccines. In infants, these were $1.95(0.44-8.72$, $p=0.38), 1.41(0.29-6.94, p=0.67)$ and $2.85(0.43-18.9, p=0.28)$, respectively. These limited data suggest no significant ITP risk following vaccinations or simultaneous vaccination in any age group, including infants.
\end{abstract}

Keywords Immune thrombocytopenic purpura $\cdot$ Inactivated vaccines - Adverse reaction $\cdot$ Simultaneous administration · Infant

\section{Introduction}

Immune thrombocytopenic purpura (ITP) is a haemorrhagic disorder characterised by thrombocytopenia, a purpuric rash, normal bone marrow and the absence of signs of other identifiable causes of thrombocytopenia [1-5]. Previous studies have shown that ITP risk increases after measles, rubella, chickenpox and influenza infection [2, 6-8]. Recently, live measles-mumps-rubella (MMR) [8-11] and varicella [12] vaccines have been reported to potentially increase the risk for ITP. Studies have also suggested that inactivated hepatitis

Electronic supplementary material The online version of this article (https://doi.org/10.1007/s12185-020-02866-1) contains supplementary material, which is available to authorized users.

Hiroshi Yokomichi

hyokomichi@yamanashi.ac.jp

Extended author information available on the last page of the article
B [13] and diphtheria-tetanus-acellular pertussis (DTaP) [12] vaccines may increase ITP risk.

Evidence suggests that ITP risk after vaccination increases through the same mechanism as that by which microbial infections induce antiplatelet autoantibodies [14]. Because vaccines are designed to induce protective immunity by mimicking infections in the human body, both live and inactivated vaccinations can theoretically trigger the development of ITP [15]. Researchers have investigated associations between multiple vaccinations and ITP risk, but findings regarding inactivated vaccinations were inconsistent [16-20]. However, because many vaccines are administered to children in infancy, an epidemiological study for vaccine safety conducted some time after a single vaccine dose is difficult to design. Therefore, more studies are needed to clarify the ITP risk associated with live and inactivated vaccines.

A combined vaccine is defined as a single product in which equivalent component vaccines are administered as a single vaccine to prevent more than one disease or to protect against multiple strains of infectious microbes [21]. For 
example, MMR, diphtheria-pertussis-tetanus and multivalent pneumococcal conjugate vaccines have been licensed for large-scale supply. The nature of combined vaccines means it is difficult to separately measure the adverse reaction rate of each component.

Simultaneous vaccination, which is commonly conducted for children in Europe [22], North America [23, 24] and Asia [25], is defined as administering more than one vaccine at different anatomic sites during the same clinic visit, without combining these vaccines in the same syringe [21]. Early research reported that parents were concerned about the efficacy of simultaneous immunisation [26]. However, experimental and clinical epidemiological studies provided a scientific basis for the efficacy of this practice [27]. Recently, the focus of interest in simultaneous vaccination has moved from questions of efficacy to questions of safety [28-30]. If a vaccination has adverse reaction risks, these risks may accumulate in simultaneous vaccination [31]. However, no research has compared the risks when administering vaccines simultaneously versus separately in the same population.

Studies conducted in the 1990s indicated rates of adverse reactions were similar between simultaneous and separate vaccine administration [32-34]. Correspondingly, the Centers for Disease Control and Prevention issued a guideline recommending that parents use simultaneous vaccination for children younger than 2 years to avoid missing the appropriate vaccination timing [21]. However, some studies suggested that rates of several adverse reactions may increase with simultaneous vaccination $[27,35]$. Therefore, the risk associated with simultaneous vaccine administration needs to be investigated more comprehensively.

In this case-control study, we aimed to determine the ITP risk after live, inactivated and simultaneous vaccination in Japan. We also examined the risk associated with simultaneous vaccination among subjects aged $<2$ years, referred to in this study as infants [36], because this age group are frequently immunised using this method.

\section{Materials and methods}

\section{Enrolment}

We requested physicians from paediatrics and internal medicine departments at seven university hospitals and 18 regional centre hospitals in Japan to recruit inpatients and outpatients for this study. Recruitment occurred from 1 October 2015 to 27 March 2017. During this period, participating physicians enrolled in all new cases with ITP that attended their hospital and met the inclusion criteria for this study. These physicians filled in the study questionnaire with the requested data for the case and control patients.

\section{Vaccines and other measurements}

We defined exposure as vaccination within 28 days before the onset of ITP. To measure this exposure, participating physicians who treated case (ITP) and control (other diseases) patients completed questionnaires covering retrospective information on vaccination history and other characteristics. In Japan, vaccination history for infants and preschool children is recorded in the Maternal and Child Health Handbook, which is provided by the municipality. Vaccination history for other children and adults was captured in a medical interview conducted by physicians or obtained from medical records. Additional information was obtained from hospital medical records. In Japan, the Immunization Law defines ITP as an adverse event following immunisation that occurs within 28 days after vaccine administration [37]. Based on this law, we considered $\leq 28$ days after administration as the duration in which vaccinated people could potentially develop ITP as a result of vaccination.

The live vaccines investigated were the bacillus Calmette-Guérin (BCG), rotavirus, varicella, mumps and measles-rubella (MR) vaccines (in Japan, MR and mumps vaccines are administered separately). Inactivated vaccines were the influenza, diphtheria-pertussis-tetanus-polio (DPT-IPV), hepatitis B, 13-valent pneumococcal conjugate, Haemophilus influenzae type b (Hib) and Japanese encephalitis vaccines. Manufacturers of vaccines administered in Japan are Daiichi Sankyo Company Ltd.; Takeda Pharmaceutical Company Ltd.; KM Biologics Company Ltd.; Biken Company Ltd.; Denka Seiken Company Ltd.; Japan BCG Laboratory; Merck Sharp \& Dohme Corp.; Sanofi S.A.; Pfizer Inc.; and GlaxoSmithKline K.K.

\section{Cases and controls}

Following Japanese and American guidelines [38-41], cases with ITP were identified when patients met all of the following conditions. (1) Peripheral blood platelet count $\leq 100,000 / \mu \mathrm{L}$ [41-43]. (2) Without anaemia unless the patient was bleeding as a result of ITP. (3) Without deformation of red or white blood cells. (4) Without aplastic anaemia, myelodysplastic syndrome, leukaemia, malignant lymphoma, paroxysmal nocturnal haemoglobinuria, systemic lupus erythematosus, disseminated intravascular coagulation, bone marrow metastasis, myelofibrosis, thrombotic thrombocytopenic purpura, haemolytic uraemic syndrome, liver cirrhosis, hypersplenism and megaloblastic anaemia. (5) Without Bernard-Soulier, Wiskott-Aldrich, May-Hegglin and Kasabach-Merrit syndromes. (6) Condition was not pseudo-thrombocytopenia 
due to ethylenediaminetetraacetic acid. (7) Condition was not thrombocytopenia caused by pharmacological agents, radiation or measles. Because this study included patients with new onset ITP, all ITP cases were considered to be as acute [42].

Each patient with ITP (case) was matched with one or two control patients [29, 30]. Participating physicians matched controls with case patients by the institution, timing of hospital visit (within a 1-month difference), sex and age. In the matched study design, we permitted overlapping use of case patient datum to be paired with two different control patient data; within an institution, one control datum was paired with two different case patient data. We requested that participating physicians reduced the difference in timing of hospital visit between case and control patients to within 2 weeks where possible. Age was matched as age in months for case patients aged under 1 year, and within 2 years for case patients aged 1-17 years. For case patients aged $\geq 18$ years, age was matched within 2 years where possible, with a maximum difference of 5 years. All participating patients were of Asian ethnicity. Sex, age at hospital visit and consulted department were measured; height and body weight were not measured.

\section{Statistical analysis}

To exploit the matched study design, we calculated McNemar's odds ratios (ORs) and their 95\% confidence intervals (CIs) [44] for the primary outcome. McNemar's OR is suitable for matched case-control design. Briefly, in this calculation method, vaccination histories for case-control pairs were grouped into four profiles: $(1)$ vaccination $(+)$ for a patient with immune thrombocytopenic purpura (case) and vaccination $(+)$ for a patient with other diseases (control); $(2)$ vaccination $(+)$ for case and vaccination $(-)$ for control; (3) vaccination ( - ) for case and vaccination $(+)$ for control; and (4) vaccination ( - ) for case and vaccination (-) for control. In calculating McNemar's ORs, only two of these profiles ( 2 and 3 ) were used based on an assumption of the binary distribution. For example, if the number of the case-control vaccination profile 2 equalled that of profile 3, McNemar's OR $=1$ (null hypothesis) [45]. If the former number of profile 2 doubled the latter number of profile $3, \mathrm{McNemar}$ 's $\mathrm{OR}=2$. This calculation method originated when McNemar's OR was developed to evaluate the results of a matched case-control study. Subsequently, conditional logistic regression was developed to further adjust for covariates.

We also calculated McNemar's ORs stratified by age group. Because vaccination for measles, rubella and varicella are known risk factors for ITP onset [8-12], we calculated those ORs as the reference values. Tests for $p$ values were based on a binary distribution with the null hypothesis being the same pair of numbers between the two vaccination history profiles, which is usually used in calculating NcNemar's OR [44].

We also calculated common ORs to exploit all available data for unmatched case and control patients. Data for those patients whose paired partner was excluded from the matched data analysis were included in the unmatched analysis. Common ORs were the secondary outcome. We aimed to measure confounders of history of infection with helicobacter pylori $[42,46]$, other viruses and bacteria, but only obtained these data for a subset of patients. Therefore, in estimating common ORs, we only adjusted for sex [10, $47,48]$, and calculated ORs stratified by age group. The age groups were $<2,2-5,6-17$ and $\geq 18$ years, based on the vaccination schedule for children, adults and older adults recommended by the Japan Pediatric Society and the Infectious Disease Surveillance Center (English versions) [25, 49].

Because the risk associated with simultaneous vaccination could not be assessed in the matched analysis because of the small sample size, this risk was only assessed in the unmatched analysis. We calculated sex-adjusted common ORs for ITP occurrence in the simultaneous administration of two or more vaccines. We performed all statistical analyses using SAS version 9.4 (SAS Institute, Cary, NC, USA). All reported $p$ values were two-sided, and $p<0.05$ was considered significant.

\section{Results}

\section{Participants}

In total, 114 pairs were included in the matched analyses, and 175 patients in the unmatched analyses. Most patients with ITP were enrolled from paediatrics departments. In the ITP case and control groups, varied infectious diseases were reported 1 month before the hospital visit. There were no underlying diseases involving an immunocompromised status, but there was an epilepsy case in the ITP case group and an asthma case in the control group. Table 1 shows the characteristics of the matched case and control patients. In total, 64 participants $(36.5 \%)$ were under 2 years of age, 52 (29.7\%) were aged 2-5 years and $76(43.4 \%)$ were male. For the 16 cases on whom data was provided by their physicians, the mean (standard deviation) of duration from vaccination to ITP onset was 17.4 (11.1) days. Among case patients, there were 18 cases of purpuras, three of bleeding in the oral cavity and difficulty stopping bleeding, three of hypermenorrhoea and one of haematochezia. Diagnoses recorded for hospital visits among control patients included 16 respiratory tract infections, nine digestive tract infections, three urinary tract infections, nine allergic diseases and four malignant neoplasms. There were 16 positive results for any 
Table 1 Characteristics of matched immune thrombocytopenic purpura case and control patients in this case-control study

\begin{tabular}{lll}
\hline Characteristic & Case patients $(n=62)$ & Control patients $(n=113)$ \\
\hline Platelet count ${ }^{\dagger}$, mean (SD) & $10,500(12,577)$ & $299,608(100,415)$ \\
Platelet count ${ }^{\dagger}$, median (interquartile range) & $6000(3000,15,000)$ & $294,000(233,000,357,000)$ \\
Male sex, $n(\%)$ & $27(44)$ & $49(43)$ \\
Age group, $n(\%)$ & & \\
$<2$ years & $22(35)$ & $42(37)$ \\
2-5 years & $19(31)$ & $33(29)$ \\
6-11 years & $12(19)$ & $22(19)$ \\
12-17 years & $1(2)$ & $2(2)$ \\
$\geq 18$ years & $8(13)$ & $14(12)$ \\
Consulted department, $n(\%)$ & & \\
Paediatrics & $54(87)$ & $99(88)$ \\
Haematology & $8(13)$ & $12(11)$ \\
Respiratory disease & 0 & $2(2)$ \\
Number of vaccinations ${ }^{\ddagger}$ & & \\
Bacillus Calmette-Guérin & 2 & 0 \\
Rotavirus & 1 & 2 \\
Varicella & 0 & 4 \\
Mumps & 0 & 1 \\
Measles-rubella & 2 & 1 \\
Influenza & 6 & 7 \\
Diphtheria-pertussis-tetanus-polio & 5 & 7 \\
Hepatitis B & 3 & 2 \\
13-valent pneumococcal conjugate & 0 & 5 \\
Haemophilus influenzae & 1 & \\
Japanese encephalitis & & \\
\hline
\end{tabular}

$S D$ standard deviation

${ }^{\dagger}$ The platelet count data for some participants was not provided by their physicians. The data shown here are from the 159 subjects for whom we have these values

${ }^{\ddagger}$ In several instances, numbers of vaccination among matched individuals differed from those of unmatched individuals in the Supplementary Tables helicobacter pylori test in the case group, but no positive results in the control group. Figure 1 presents a histogram of the ages of case patients. Most case patients with ITP were aged $<1-14$ years.

\section{Pairwise analyses}

Table 2 shows the McNemar's ORs, stratified by live/inactivated vaccine and age group. For all age groups, the $\mathrm{McNe}-$ mar's OR for ITP occurrence was 1.80 (95\% CI 0.54-6.84, $p=0.64)$ for all vaccines; 1.00 (95\% CI 0.19-5.37, $p=0.50)$ for live vaccines; 2.33 (95\% CI 0.53-14.0, $p=0.70$ ) for inactivated vaccines; and 2.00 (95\% CI $0.03-157, p=1.00$ ) for the MR vaccine. The OR for the varicella vaccine could not be calculated because of an unbalanced number combination in the four vaccination history profiles of case-control pairs. In infants aged under 2 years, the McNemar's ORs for ITP occurrence in cases for all vaccines, live vaccines and inactivated vaccines were 1.50 (95\% CI $0.17-18.0, p=0.50), 2.00$
(95\% CI 0.29-22.1, $p=0.67)$ and 1.00 (95\% CI 0.01-78.5, $p=0.50$ ), respectively. Supplementary Tables $1-3$ present cross-tabulations of the four profiles of matched pairs for all, live and inactivated vaccines.

\section{Non-pairwise analyses}

Table 3 shows the sex-adjusted common ORs stratified by live/inactivated vaccine and age group. The common OR for ITP occurrence was 1.39 (95\% CI 0.61-3.14, $p=0.50)$ for all vaccines, 2.18 (95\% CI $0.53-9.40, p=0.34)$ for live vaccines, 1.28 (95\% CI $0.52-3.08, p=0.69)$ for inactivated vaccines and 3.55 (95\% CI 0.18-216, $p=0.60$ ) for the MR vaccine. The OR for the varicella vaccine could not be calculated because of the small number of ITP cases in this exposure. In infants, the common ORs for ITP occurrence in cases for all vaccines, live vaccines and inactivated vaccines were 1.43 (95\% CI 0.43-4.68, $p=0.68$ ), 4.26 (95\% CI $0.80-29.3, p=0.10)$ and 0.99 (95\% CI $0.26-3.55, p=1.00$ ), 
Histograms of ages of patients with ITP (cases)

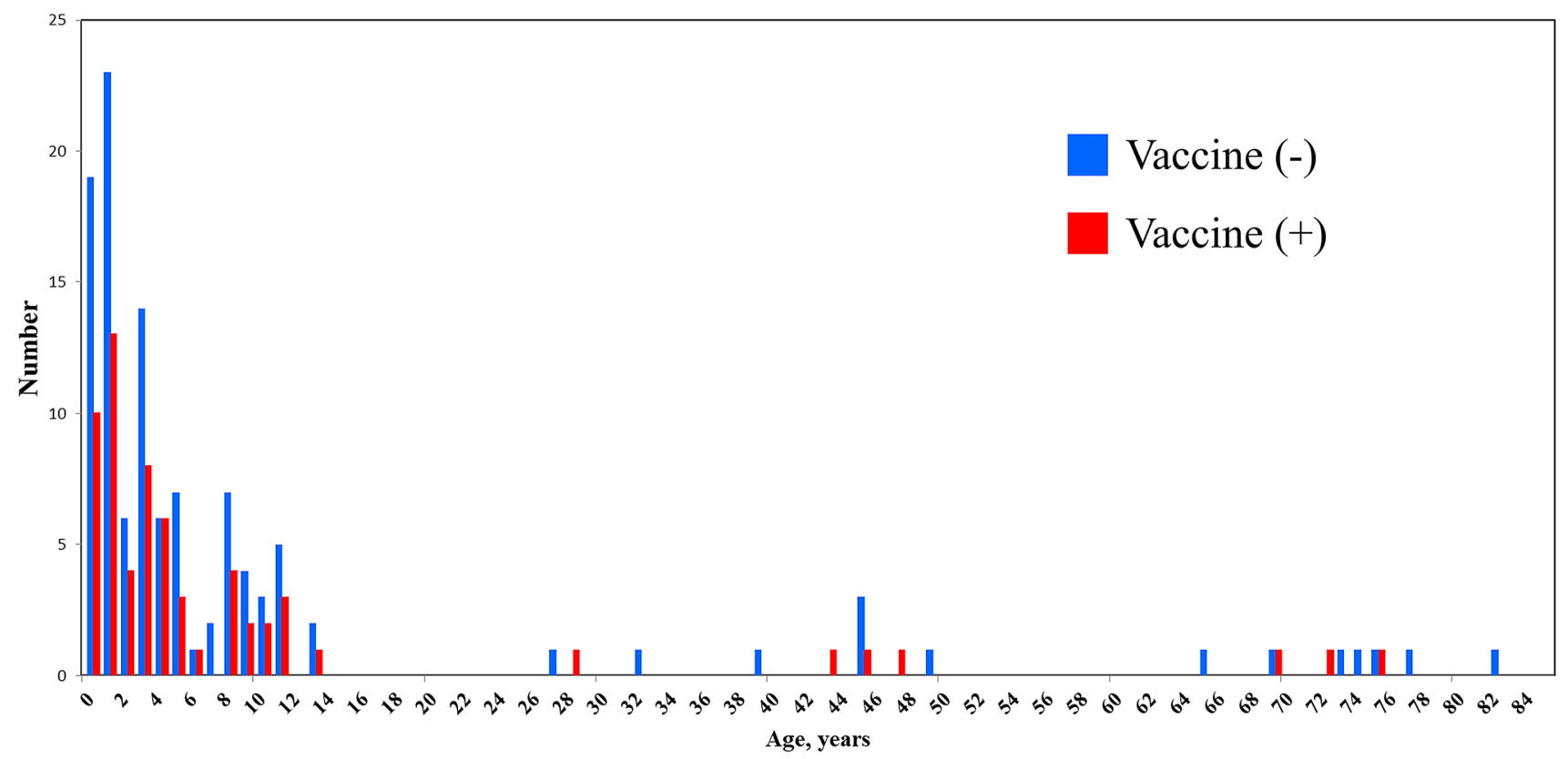

Fig. 1 Histogram of ages among case patients with immune thrombocytopenic purpura

Table 2 McNemar's odds ratios (95\% confidence intervals) for immune thrombocytopenic purpura occurrence after vaccination by age group

\begin{tabular}{lcll}
\hline $\begin{array}{l}\text { Age } \\
\text { group } \\
\text { (years) }\end{array}$ & All vaccinations & Live vaccination & $\begin{array}{l}\text { Inactive vac- } \\
\text { cination }\end{array}$ \\
\hline All & $1.80(0.54-6.84)$ & $1.00(0.19-5.37)$ & $2.33(0.53-14.0)$ \\
$<18$ & $2.25(0.63-10.00)$ & $1.00(0.19-5.37)$ & $2.33(0.53-14.0)$ \\
$<2$ & $1.50(0.17-18.0)$ & $2.00(0.29-22.1)$ & $1.00(0.01-78.5)$ \\
$2-5$ & $0.50(0.01-9.60)$ & Unable to estimate & $0.50(0.01-9.60)$ \\
\hline
\end{tabular}

Odds ratios for age group for $\geq 18$ years and 6-17 years could not be estimated because of the small sample sizes

respectively. The common OR for inactivated vaccines was significantly high among children aged 6-17 years (OR 13.6, 95\% CI $1.23-747, p=0.028$ ). Supplementary Tables $4-21$ present the cross-tabulations of individual vaccines for the unmatched data. The numbers of case and control patients differed between pairwise and non-pairwise analyses.

\section{Simultaneous vaccination}

For simultaneous vaccination, we investigated children aged 2 months to 11 years: six children had two vaccines, four had three vaccines, two had four vaccines and one had five vaccines. Table 4 shows the sex-adjusted common ORs for ITP occurrence in the simultaneous administration of two or more vaccines. The ORs were 1.52 (95\% CI 0.45-5.21, $p=0.71)$ in children aged $<12$ years and $1.95(95 \% \mathrm{CI}$ $0.44-8.72, p=0.28$ ) in children aged $<2$ years. The ORs for the simultaneous administration of inactivated vaccines were 1.83 (95\% CI $0.44-7.59, p=0.40)$ in children of all ages and $1.41(95 \%$ CI $0.29-6.94, p=0.67)$ in children aged $<2$ years.
Table 3 Sex-adjusted odds ratios $(95 \%$ confidence intervals) for immune thrombocytopenic purpura occurrence after vaccination by age group

\begin{tabular}{lllll}
\hline $\begin{array}{l}\text { Age group } \\
\text { (years) }\end{array}$ & All vaccinations & MR vaccination & Live vaccination & Inactivated vaccination \\
\hline All & $1.39(0.61-3.14)$ & $3.55(0.18-216)$ & $2.18(0.53-9.40)$ & $1.28(0.52-3.08)$ \\
$<18$ & $1.60(0.68-3.75)$ & $3.53(0.18-216)$ & $2.19(0.53-9.50)$ & $1.38(0.55-3.42)$ \\
$<2$ & $1.43(0.43-4.68)$ & $3.49(0.17-225)$ & $4.26(0.80-29.3)$ & $0.99(0.26-3.55)$ \\
$2-5$ & $0.38(0.01-4.11)$ & Unable to estimate & Unable to estimate & $0.38(0.01-4.11)$ \\
$6-17$ & $13.6(1.23-747)$ & Unable to estimate & Unable to estimate & $13.6(1.23-747)$ \\
\hline
\end{tabular}

Odds ratios adjusted for sex

$M R$ measles-rubella 
Table 4 Sex-adjusted odds ratios (95\% confidence intervals) for immune thrombocytopenic purpura occurrence in simultaneous administration of vaccines among children aged $<12$ years

\begin{tabular}{llllll}
\hline $\begin{array}{l}\text { Age group } \\
\text { (years) }\end{array}$ & Single vaccination & $\begin{array}{l}\text { All simultaneous vac- } \\
\text { cinations }\end{array}$ & $\begin{array}{l}\text { Simultaneous live } \\
\text { vaccinations }\end{array}$ & $\begin{array}{l}\text { Simultaneous inactivated } \\
\text { vaccinations }\end{array}$ & $\begin{array}{l}\text { Simultaneous live } \\
\text { and inactivated } \\
\text { vaccinations }\end{array}$ \\
\hline$<12$ & $1.38(0.57-3.33)$ & $1.52(0.45-5.21)$ & Unable to estimate & $1.83(0.44-7.59)$ & $1.36(0.29-6.30)$ \\
$<2$ & $1.21(0.34-4.34)$ & $1.95(0.44-8.72)$ & Unable to estimate & $1.41(0.29-6.94)$ & $2.85(0.43-18.9)$ \\
\hline
\end{tabular}

${ }^{\dagger}$ Odds ratios for simultaneous live vaccinations could not be estimated because of small sample sizes

${ }^{\ddagger}$ Simultaneous administration of live and inactivated vaccines

The ORs for the simultaneous administration of live and inactivated vaccines were $1.36(95 \%$ CI $0.29-6.30, p=0.69)$ in children of all ages and $2.85(95 \%$ CI $0.43-18.9, p=0.28)$ in children aged $<2$ years.

\section{Discussion}

\section{Statement of principal findings}

The McNemar's and common ORs for ITP occurrence for all, live and inactivated vaccines ranged from almost null to more than 1 among people of all ages, children aged $<18$ years and children aged $<2$ years, but these results were not statistically significant. The common ORs for simultaneous administration of two or more vaccines were greater than 1 for all, live and inactivated vaccines; these results were not statistically significant.

\section{Results in the context of previous reports}

Previous research suggests that people may develop ITP as an immune reaction to vaccination in combination with a genetic predisposition [4, 50-54]. Because vaccines are designed to mimic real infections and trigger immune reactions, clinicians have been concerned that vaccinationparticularly the administration of live vaccines-may cause autoimmune disease [50]. MMR-associated acute ITP has been frequently investigated in studies on this topic [9, 51-53]. In 1994, the United States Institute of Medicine acknowledged that evidence had established a causal relationship between MMR vaccination and thrombocytopenia [55]. In our study, the common OR for MR vaccination was 3.55 , although this result was not statistically significant (Supplementary Table 15). In summary, the present study did not find many significant effects, but the McNemar's and common ORs estimated for live vaccination ranged from null to high across age groups.

ITP risk associated with inactivated vaccination has not been sufficiently investigated in previous studies. A postlicensure retrospective study in the United States did not find any ITP cases among 2-month-old infants vaccinated with the DTaP-IPV/Hib or other DTaP-containing vaccine from 1 October 2008 to 31 July 2010 [17]. A Canadian surveillance study (1992-2001) identified eight ITP cases after DTP or $\mathrm{DTaP} \pm \mathrm{IPV} \pm$ Hib vaccinations, three after hepatitis B vaccination and two after influenza vaccination in 12 paediatric hospitals [18]. An Italian post-licensure prospective study involving a large number of older adults conducted from 2006 to 2009 reported 13 cases of ITP after seasonal influenza vaccinations [19]. A study using a Taiwanese national database found an incidence rate ratio for ITP of 1.09 (95\% CI 0.65-1.85) following pandemic influenza (H1N1) 2009 monovalent vaccination without adjuvant versus non-vaccination among people aged $0-42$ years, and of 1.05 (95\% CI 0.41-2.85) among children [20]. The United States Vaccine Adverse Event Reporting System database reported that from 1990 to 2008, influenza vaccination had a proportional reporting ratio (an index of identifying unexpectedly frequent reports) for thrombocytopenia occurrence of 2.10 (17 cases, lower bound of $90 \% \mathrm{CI}$ of the empirical Bayesian geometric mean <2.0) [16]. However, there is a lack of consistent evidence about the ITP risk associated with inactivated vaccines.

In our study, the estimated McNemar's ORs for inactivated vaccines varied from under 1 to over 1 , and were not statistically significant in the examined age groups. Calculation of McNemar's ORs used discordant case-control pairs in terms of vaccination history; therefore, calculating $\mathrm{McNe}$ mar's ORs required a much larger sample size than would have been required to calculate common ORs [56]. Because of insufficient power, we were unable to clarify whether ITP risk accompanied inactivated vaccination.

\section{Contributions to clinicians and parents}

Care should be taken in the interpretation of the ORs reported for inactivated vaccines in this study. In the context of Japan, many inactivated vaccinations are scheduled for children aged $<1$ year [25], meaning that infants of this age have a high chance of being immunised with inactivated vaccines. Previous research suggests that among Japanese children, the peak age of ITP onset is $<2$ years [48]. The ORs for inactivated vaccination could, therefore, 
be overestimated in this study because most data used for their estimation included data for infants aged $<1$ year, who frequently receive inactivated vaccines and have a relatively high incidence of ITP. Therefore, we interpreted the McNemar's OR of 1.00 (Table 2) and common OR of 0.99 (Table 3 ) among children aged $<2$ years as indicating that this study did not detect ITP risk in inactivated vaccination.

Vaccination is frequently criticised in the media [29, 30]. However, vaccination has three types of benefits: direct effects (immunity of the vaccinated person), indirect effects (herd protection of non-vaccinated people) and reduction in the risk for developing ITP caused by infection with viruses or bacteria. The third type of benefit could be rephrased as the observation that the incidence of ITP following vaccination is lower than the incidence of ITP after infection with wild viruses [50]. The incidence of ITP development in children immunised with MMR is reportedly around one in 30,000, whereas it is approximately one in 3000 following natural rubella infection and approximately one in 6000 following measles infection. Another report suggested that the incidence of ITP caused by MMR vaccination is 10-20 times lower than the incidence of ITP following natural infection [6]. As is inherent with a single outcome (in this case, the development of ITP), the present findings of ORs greater than 1 do not consider the third type of vaccination benefit. In other words, it is difficult to investigate the long-term effect of vaccination on preventing ITP development from the perspective of the third type of benefit - if it exists—in cohort or case-control studies.

The present study is notable for investigating ITP risk following simultaneous vaccination. Although we did not find statistically significant results, the sex-adjusted ORs were relatively high (OR 1.52 among children aged $<12$ years and 1.95 among children aged $<2$ years; Table 3). The point estimate of the OR was larger than that of single vaccination ( 1.38 among children aged $<12$ years and 1.21 among children aged $<2$ years; Table 3 ) in children of each age group. Few studies have investigated the risks associated with simultaneous vaccination. A randomised controlled trial involving people receiving simultaneous influenza vaccination reported increased haemagglutinin inhibition titre, seroconversion and seropositivity rates, suggesting that the immune response was boosted [57]. Because vaccine efficacy and immune-related adverse reactions have the same origin [15], the rate of ITP development may increase depending on the administration method. Table 4 suggests that there is potential for a relatively higher risk for ITP with simultaneous vaccination compared with separate vaccination. However, further analyses using large databases are needed to confirm this finding.

\section{Limitations and strengths}

There are several limitations inherent to this study. First, the sample size meant the study was underpowered because we adopted a matched design to adjust for several confounders between cases and controls. This sample size also made it difficult to estimate ORs separately by age group. To compensate for this limitation and present additional results, we calculated common ORs using all available data, although adjustment for confounders might have been insufficient for this analysis. Second, we could not adjust for infection history as a confounder. Although we aimed to measure infection history in the questionnaire, we did not obtain sufficient data. Third, because the investigation depended on the voluntary replies of physicians by post, reporting bias might have occurred. Physicians who were interested in potential adverse events might have been more likely to participate in this study, which could have led to an overestimation of the ORs. A national surveillance system of adverse events following vaccinations in Japan is needed in an era when people are concerned about drug-induced diseases. Fourth, each case was matched to one or two controls. This method might have resulted in an imbalance in the weight of samples for the primary outcome. Because several matching factors may restrict selecting potential control patients, we chose a design to counter this limitation.

In terms of study strengths, the detection of ITP cases by physicians was a main advantage of the present study. The use of physician-registered cases enhanced the internal validity of this investigation. This validity would not be possible in a large database study. Second, the data were gathered from hospitals across Japan. The reported ITP risks, therefore, reflect a broad area, suggesting relatively high external validity. Third, the analyses included detailed results stratified by age group and type of vaccine. This information will be informative for researchers and clinicians seeking to assess the potential risks for adverse events for their patients.

\section{Conclusions}

Based on the limited data available in this study, high ITP risk was not found following inactivated or simultaneous vaccination. Future investigations of ITP risk should include analyses using large databases.

Acknowledgements We would like to express our deep gratitude to the participating patients. For their cooperation as physicians in this study, we would also like to thank Dr. Katsuko Maeda, Yamagata City Hospital Saiseikan; Dr. Yuya Sato, Dokkyo Medical University Hospital; Dr. Noritaka Furuya, Saitama Citizens Medical Centre; Dr. Yoshinori Kobayashi, National Hospital Organization Yokohama Medical Centre; 
Dr. Akira Nakayama, Saiseikai Yokohamashi Tobu Hospital; Eriko Nakazawa, Yokohama Minami Kyosai Hospital; Dr. Yasuhiro Igawa, Kanazawa University Hospital; Dr. Shigeki Hotta, Ishikawa Prefectural Central Hospital; Dr. Tetsuo Otsuki, Komatsu Municipal Hospital; Dr. Harue Tsuji, Takaoka City Hospital; Dr. Akihiko Tanizawa, University of Fukui Hospital; Dr. Yoshihiro Taniguchi, Japanese Red Cross Fukui Hospital; Dr. Tomoyuki Nakazawa, Toshima Hospital; Dr. Tomohiro Saito, Yamanashi Prefectural Central Hospital; Dr. Koji Kobayashi, Yamanashi Kosei Hospital; Dr. Mie Mochizuki, University of Yamanashi Hospital; Dr. Ryosuke Yamamura, Osaka Saiseikai Nakatsu Hospital; Dr. Tomoko Takano, Osaka General Medical Centre; Dr. Asao Hirose, Osaka City University; Dr. Kiryan Ko, Osaka General Hospital of West Japan Railway Company; Dr. Takaaki Tanaka, Kawasaki Medical School General Medical Center; Drs. Hideto Teranishi and Kenichi Inamura, Kawasaki Medical School Hospital; Dr. Tateharu Urayama, Okayama Medical Centre; Dr. Keita Matsubara, Hiroshima City Funairi Citizens Hospital; Dr. Rie Shiroyama, Hospital of the University of Occupational and Environmental Health; and Dr. Hirofumi Koga, Beppu Medical Centre. We thank Jennifer Barrett, PhD and Audrey Holmes, MA, for editing drafts of this manuscript.

Author contributions KTT and ZY conceived the study. KTT, ZY, HY, RK, TN, YY, MMori and SM contributed to the study design. HY, KTT, RK, TN, YY, MMori, YA, AM, HO, HS, SA, MMochizuki and $\mathrm{ZY}$ interpreted the data and contributed to the discussion. HY analysed the data. HY, KTT, MMochizuki, TN, RK and ZY wrote the first draft of the manuscript. HY, KTT, TN, RK and MMochizuki contributed to the revision of the manuscript. KTT was responsible for data integrity. KTT and HY obtained funding. All authors agreed with the results and conclusion of the study and approved the final version of the manuscript.

Funding This work was supported by the Japan Agency for Medical Research and Development (grant numbers: 15mk0101005h0102 and 16mk0101012j0003) and the Ministry of Education, Culture, Sports, Science and Technology (grant number: JP18K17376). The funding sources had no role in study design, data collection, data analysis, data interpretation, or writing of the report.

Availability of data and materials The original data are available from the National Institute of Infectious Diseases with permission from Dr. Tanaka-Taya.

\section{Compliance with ethical standards}

Conflict of interest TN received honoraria from Daiichi Sankyo Co., Sanofi K.K. and Mitsubishi Tanabe Pharma Corporation. MMochizuki received honoraria from Pfizer Inc. MMori's department received unrestricted research grants from AbbVie GK; Ayumi Pharmaceutical Corporation; Chugai Pharmaceutical Co., Ltd.; CSL Behring K.K.; Japan Blood Products Organization; Nippon Kayaku Co., Ltd.; UCB Japan Co., Ltd.; and Asahikasei Pharmaceutical Corporation. The other authors have no conflicts of interest to declare.

Ethics approval The study protocol was reviewed and approved by the Ethics Committee of the National Institute of Infectious Diseases (the principal institution of this project) in accordance with the ethical guidelines and regulations of the Declaration of Helsinki (approval number: H27-561).

Informed consent Physicians in hospitals explained the study to their patients and obtained written informed consent from the parent(s) or legal guardian(s) of each child and/or assent by the child (when applicable) before initiating medical interview and record review.

\section{References}

1. Chu YW, Korb J, Sakamoto KM. Idiopathic thrombocytopenic purpura. Pediatr Rev. 2000;21(3):95-104.

2. Hamiel U, Kventsel I, Youngster I. Recurrent immune thrombocytopenia after influenza vaccination: a case report. Pediatrics. 2016;138(6):e20160124.

3. Oka S, Ono K, Nohgawa M. Prediction of response to first-line therapy with ITP by flow cytometric analysis of bone marrow lymphocyte phenotypes. Int J Hematol. 2020. https://doi.org/10.1007/ s12185-020-02847-4.

4. Matsumoto M, Fujimura Y, Wada H, Kokame K, Miyakawa Y, Ueda $Y$, et al. Diagnostic and treatment guidelines for thrombotic thrombocytopenic purpura (TTP) 2017 in Japan. Int J Hematol. 2017;106(1):3-15.

5. Poston JN, Gernsheimer TB. Glucocorticoids promote response to thrombopoietin-receptor agonists in refractory ITP: a case series. Int J Hematol. 2019;110(2):255-9.

6. Cines DB, Bussel JB, Liebman HA, Luning Prak ET. The ITP syndrome: pathogenic and clinical diversity. Blood. 2009;113(26):6511-21.

7. Yenicesu İ, Yetgin S, Özyürek E, Aslan D. Virus-associated immune thrombocytopenic purpura in childhood. Pediatr Hematol Oncol. 2002;19(6):433-7.

8. Bertuola F, Morando C, Menniti-Ippolito F, Da Cas R, Capuano A, Perilongo $\mathrm{G}$, et al. Association between drug and vaccine use and acute immune thrombocytopenia in childhood. Drug Saf. 2010;33(1):65-72.

9. Cecinati V, Principi N, Brescia L, Giordano P, Esposito S. Vaccine administration and the development of immune thrombocytopenic purpura in children. Hum Vaccin Immunother. 2013;9(5):1158-62.

10. France EK, Glanz J, Xu S, Hambidge S, Yamasaki K, Black $\mathrm{SB}$, et al. Risk of immune thrombocytopenic purpura after measles-mumps-rubella immunization in children. Pediatrics. 2008;121(3):e687-e692692.

11. Black C, Kaye JA, Jick H. MMR vaccine and idiopathic thrombocytopaenic purpura. Br J Clin Pharmacol. 2003;55(1):107-11.

12. Rinaldi M, Perricone C, Ortega-Hernandez O, Perricone R, Shoenfeld Y. Immune thrombocytopaenic purpura: an autoimmune cross-link between infections and vaccines. Lupus. 2014;23(6):554-67.

13. Meyboom RHB, Fucik H, Edwards IR. Thrombocytopenia reported in association with hepatitis B and A vaccines. Lancet. 1995;345(8965):1638. https://www.thelancet.com/pdfs/journals/ lancet/PIIS0140-6736(95)90143-4.pdf.

14. Schattner A. Consequence or coincidence?: The occurrence, pathogenesis and significance of autoimmune manifestations after viral vaccines. Vaccine. 2005;23(30):3876-86.

15. Perricone C, Ceccarelli F, Nesher G, Borella E, Odeh Q, Conti $\mathrm{F}$, et al. Immune thrombocytopenic purpura (ITP) associated with vaccinations: a review of reported cases. Immunol Res. 2014;60(2-3):226-35.

16. Woo EJ, Wise RP, Menschik D, Shadomy SV, Iskander J, Beeler J, et al. Thrombocytopenia after vaccination: case reports to the US Vaccine Adverse Event Reporting System, 1990-2008. Vaccine. 2011;29(6):1319-23.

17. Hansen J, Timbol J, Lewis N, Pool V, Decker MD, Greenberg DP, et al. Safety of DTaP-IPV/Hib vaccine administered routinely to infants and toddlers. Vaccine. 2016;34(35):4172-9.

18. Jadavji T, Scheifele D, Halperin S. Thrombocytopenia after immunization of Canadian children, 1992 to 2001. Pediatr Infect Dis J. 2003;22(2):119-22.

19. Villa M, Black S, Groth N, Rothman KJ, Apolone G, Weiss NS, et al. Safety of MF59-adjuvanted influenza vaccination in 
the elderly: results of a comparative study of MF59-adjuvanted vaccine versus nonadjuvanted influenza vaccine in Northern Italy. Am J Epidemiol. 2013;178(7):1139-45.

20. Huang WT, Yang HW, Liao TL, Wu WJ, Yang SE, Chih YC, et al. Safety of pandemic (H1N1) 2009 monovalent vaccines in Taiwan: a self-controlled case series study. PLoS ONE. 2013;8(3):e58827.

21. Kroger AT, Duchin J, Vázquez M. General best practice guidelines for immunization. Best practices guidance of the Advisory Committee on Immunization Practices (ACIP). US Department of Health and Human Services, Centers for Disease Control and Prevention, Atlanta, GA; 2017. Available from: https://www.cdc. gov/vaccines/hcp/acip-recs/general-recs/index.html. Accessed 4 April 2020.

22. Vaccination schedules for individual European countries and specific age groups. European Centre for Disease Prevention and Control, European Union; 2020. Available from: https://www. ecdc.europa.eu/en/immunisation-vaccines/EU-vaccination-sched ules. Accessed 4 April 2020.

23. Robinson CL, Romero JR, Kempe A, Pellegrini C, Szilagyi P. Advisory committee on immunization practices recommended immunization schedule for children and adolescents aged 18 years or younger-United States, 2018. MMWR. 2018;67(5):156.

24. National Advisory Committee on Immunization. Canada's provincial and territorial routine (and catch-up) vaccination routine schedule programs for infants and children. 2020. Public Health Agency of Canada. Available from: https://www.canada.ca/en/ public-health/services/provincial-territorial-immunization-infor mation/provincial-territorial-routine-vaccination-programs-infan ts-children.html. Accessed 4 April 2020.

25. Vaccination schedule recommended by the Japan Pediatric Society. Japan Pediatric Society; 2018. Available from: https://www. jpeds.or.jp/modules/en/index.php?content_id=7. Accessed 4 April 2020.

26. Offit PA, Quarles J, Gerber MA, Hackett CJ, Marcuse EK, Kollman TR, et al. Addressing parents' concerns: do multiple vaccines overwhelm or weaken the infant's immune system? Pediatrics. 2002;109(1):124-9.

27. King GE, Hadler SC. Simultaneous administration of childhood vaccines: an important public health policy that is safe and efficacious. Pediatr Infect Dis J. 1994;13(5):394-407.

28. Smith PJ, Chu SY, Barker LE. Children who have received no vaccines: Who are they and where do they live? Pediatrics. 2004;114(1):187-95.

29. Yokomichi H, Kurihara S, Yokoyama T, Inoue E, Tanaka-Taya $\mathrm{K}$, Kono S, et al. The pandemic influenza A (H1N1) 2009 vaccine does not increase the mortality rate of idiopathic interstitial pneumonia: a matched case-control study. PLoS ONE. 2014;9(2):e88927.

30. Yokomichi H, Kurihara S, Yokoyama T, Inoue E, Tanaka-Taya $\mathrm{K}$, Kono S, et al. Safety of the influenza A (H1N1)2009 vaccine in chronic obstructive pulmonary disease: a matched case-control study. J Vaccin Vaccin. 2012;3(5):1000148.

31. Walter S, Holford T. Additive, multiplicative, and other models for disease risks. Am J Epidemiol. 1978;108(5):341-6. https://doi. org/10.1093/oxfordjournals.aje.a112629.

32. Deforest A, Long SS, Lischner HW, Girone JA, Clark JL, Srinivasan $R$, et al. Simultaneous administration of measles-mumpsrubella vaccine with booster doses of diphtheria-tetanus-pertussis and poliovirus vaccines. Pediatrics. 1988;81(2):237-46.

33. Dashefsky B, Wald E, Guerra N, Byers C. Safety, tolerability, and immunogenicity of concurrent administration of Haemophilus influenzae type b conjugate vaccine (meningococcal protein conjugate) with either measles-mumps-rubella vaccine or diphtheriatetanus-pertussis and oral poliovirus vaccines in 14-to 23-monthold infants. Pediatrics. 1990;85(4):682-9.
34. Giammanco G, Volti SL, Mauro L, Bilancia GG, Salemi I, Barone $\mathrm{P}$, et al. Immune response to simultaneous administration of a recombinant DNA hepatitis B vaccine and multiple compulsory vaccines in infancy. Vaccine. 1991;9(10):747-50.

35. Honkanen PO, Keistinen T, Kivelä S. Reactions following administration of influenza vaccine alone or with pneumococcal vaccine to the elderly. Arch Intern Med. 1996;156(2):205-8.

36. Child Development. Infants (0-1 year of age). Centers for Disease Control and Prevention; 2020. Available from: https://www.cdc. gov/ncbddd/childdevelopment/positiveparenting/infants.html. Accessed 4 April 2020.

37. Immunization Act. Ministry of Health, Labour and Welfare. 1948. Amended in 2013. Available from: http://www.japaneselawtran slation.go.jp/law/detail/?printID $=\& \mathrm{re}=01 \& \mathrm{id}=2964 \& \mathrm{vm}=02$. Accessed 4 April 2020.

38. Shirahata A, Ishii E, Eguchi H, Okawa H, Ohta S, Kaneko T, et al. Consensus guideline for diagnosis and treatment of childhood idiopathic thrombocytopenic purpura. Int J Hematol. 2006;83(1):29-38.

39. Kurata Y, Fujimura K, Kuwana M, Tomiyama Y, Murata M. Epidemiology of primary immune thrombocytopenia in children and adults in Japan: a population-based study and literature review. Int J Hematol. 2011;93(3):329-35.

40. Rodeghiero F, Stasi R, Gernsheimer T, Michel M, Provan D, Arnold DM, et al. Standardization of terminology, definitions and outcome criteria in immune thrombocytopenic purpura of adults and children: report from an international working group. Blood. 2009;113(11):2386-93.

41. Liu X-g, Bai X-c, Chen F-p, Cheng Y-f, Dai K-s, Fang M-y, et al. Chinese guidelines for treatment of adult primary immune thrombocytopenia. Int J Hematol. 2018;107(6):615-23.

42. Kashiwagi H, Kuwana M, Hato T, Takafuta T, Fujimura K, Kurata Y, et al. Reference guide for management of adult immune thrombocytopenia in Japan: 2019 Revision. Int J Hematol. 2020;111(3):329-51.

43. Langeberg WJ, Schoonen WM, Eisen M, Gamelin L, Stryker S. Thromboembolism in patients with immune thrombocytopenia (ITP): a meta-analysis of observational studies. Int J Hematol. 2016;103(6):655-64.

44. Breslow NE, Day N. Statistical methods in cancer research. Volume 1: the analysis of case-control studies. Oxford: Oxford University Press; 1980.

45. Fagerland MW, Lydersen S, Laake P. The McNemar test for binary matched-pairs data: mid-p and asymptotic are better than exact conditional. BMC Med Res Methodol. 2013;13(1):91.

46. Takase K, Nagai H, Kadono M, Yoshioka T, Yoshio N, Hirabayashi Y, et al. High-dose dexamethasone therapy as the initial treatment for idiopathic thrombocytopenic purpura. Int J Hematol. 2020;111(3):388-95.

47. Reading R. Risk of immune thrombocytopenic purpura after measles-mumps-rubella immunization in children. Child Care Health Dev. 2008;34(4):545-8.

48. Shirahata A, Fujisawa K, Ishii E, Ohta S, Sako M, Takahashi $\mathrm{Y}$, et al. A nationwide survey of newly diagnosed childhood idiopathic thrombocytopenic purpura in Japan. J Pediatr Hematol Oncol. 2009;31(1):27-322.

49. Routine/voluntary immunization schedule in Japan. Infectious Disease Surveillance Center; 2016. Available from: https://www. niid.go.jp/niid/images/vaccine/schedule/2016/EN20161001.pdf. Accessed 4 April 2020.

50. Wraith DC, Goldman M, Lambert PH. Vaccination and autoimmune disease: what is the evidence? Lancet. 2003;362(9396):1659-66.

51. Miller E, Waight P, Farrington C, Andrews N, Stowe J, Taylor B. Idiopathic thrombocytopenic purpura and MMR vaccine. Arch Dis Child. 2001;84(3):227-9. 
52. Vlacha V, Forman EN, Miron D, Peter G. Recurrent thrombocytopenic purpura after repeated measles-mumps-rubella vaccination. Pediatrics. 1996;97(5):738-9.

53. Oski FA, Naiman JL. Effect of live measles vaccine on the platelet count. N Engl J Med. 1966;275(7):352-6. https://doi.org/10.1056/ NEJM196608182750703.

54. Rab MAE, Meerveld-Eggink A, van Velzen-Blad H, van Loon D, Rijkers GT, de Weerdt O. Persistent changes in circulating white blood cell populations after splenectomy. Int J Hematol. 2018;107(2):157-65.

55. Stratton KR, Howe C, Johnston RB Jr. Adverse events associated with childhood vaccines other than pertussis and rubella: Summary of a report from the institute of medicine. JAMA. 1994;271(20):1602-5.
56. Lachenbruch PA. On the sample size for studies based upon monemar's test. Stat Med. 1992;11(11):1521-5.

57. Vajo Z, Tamas F, Sinka L, Jankovics I. Safety and immunogenicity of a 2009 pandemic influenza A H1N1 vaccine when administered alone or simultaneously with the seasonal influenza vaccine for the 2009-10 influenza season: a multicentre, randomised controlled trial. Lancet. 2010;375(9708):49-55.

Publisher's Note Springer Nature remains neutral with regard to jurisdictional claims in published maps and institutional affiliations.

\section{Affiliations}

\section{Hiroshi Yokomichi ${ }^{1}$ (1) Keiko Tanaka-Taya ${ }^{2} \cdot$ Rie Koshida $^{3} \cdot$ Takashi Nakano $^{4} \cdot$ Yoshinori Yasui $^{5} \cdot$ Masaaki Mori $^{6}$. $^{2}$ Yuka Ando ${ }^{7}$. Saeko Morino ${ }^{2} \cdot$ Hideo Okuno $^{2} \cdot$ Hiroshi Satoh $^{2} \cdot$ Satoru Arai $^{2} \cdot$ Mie Mochizuki $^{8} \cdot$ Zentaro Yamagata $^{1}$}

\author{
Keiko Tanaka-Taya \\ ktaya@niid.go.jp \\ Rie Koshida \\ koshida_r@city.kanazawa.lg.jp \\ Takashi Nakano \\ nakano@med.kawasaki-m.ac.jp \\ Yoshinori Yasui \\ yasui4900220@nakatsu.saiseikai.or.jp \\ Masaaki Mori \\ mmori@med.yokohama-cu.ac.jp \\ Yuka Ando \\ yuka700411@yahoo.co.jp \\ Saeko Morino \\ s-morino@nih.go.jp \\ Hideo Okuno \\ okuno-h@nih.go.jp \\ Hiroshi Satoh \\ satohiro@nih.go.jp \\ Satoru Arai \\ arais@nih.go.jp \\ Mie Mochizuki \\ mmie@sweet.ocn.ne.jp
}

Zentaro Yamagata

zenymgt@yamanashi.ac.jp

1 Department of Health Sciences, University of Yamanashi, 1110 Shimokato, Chuo, Yamanashi 409-3898, Japan

2 National Institute of Infectious Diseases, 1-23-1 Toyama, Shinjuku Ward, Tokyo 162-8840, Japan

3 City of Kanazawa, 1-1-1 Hirosaka, Kanazawa, Ishikawa 920-0962, Japan

4 Kawasaki Medical School General Medical Center, 2-6-1 Nakasange, Okayama, Okayama 700-8505, Japan

5 Osaka Saiseikai Nakatsu Hospital, 2-10-39 Shibata, Kita Ward, Osaka, Osaka 530-0012, Japan

6 Tokyo Medical and Dental University, 1-5-45 Yushima, Bunkyo Ward, Tokyo 113-8510, Japan

7 National Hospital Organization Iwakuni Clinical Center, 1-1-1 Atago, Iwakuni, Yamaguchi 740-8510, Japan

8 Department of Pediatrics, University of Yamanashi, 1110 Shimokato, Chuo, Yamanashi 409-3898, Japan 